\section{UPPER PAL feolithic Age}

The highly significant evidence for the Upper Palæolithic period, drawn in part from cave deposits, in part from north Lincolnshire open sites, must here be dismissed in brief. Among the more interesting conclusions to which excavation has led, is the confirmation of the absence of any gap in continuity between Upper Palæolithic and Mesolithic, and at the elose of the Aurignacian period the remarkable local variability in type of the elements of the culture. Two finds which must not be passed over are a fragment of mother-of-pearl skilfully shaped, possibly part of an amulet, and a cowrie shell, Cyprea moneta, from the Pin Hole Cave.

\title{
HEARING TESTS
}

$\mathrm{I}^{\mathrm{N}}$ the December number of the Bell Laboratories Record, Mr. H. C. Montgomery, of the Acoustical Research Department of the Bell Laboratories, discusses results obtained by subjecting many of the visitors at the World's Fairs to tests of the quality of their hearing. The records obtained enabled him to get nearly three-quarters of a million of records carried out in the same way.

The Bell system deals in hearing, and to get the best service it is important that it have available trustworthy data on the hearing characteristics of the American people. The tests are given in soundproof rooms arranged to seat seven visitors, each partially screened from the others. Both the tests and the instructions are given through telephone receivers; the visitor holds the receiver to his ear with one hand whilst he marks the results on a card with the other. Two types of tests are given, with separate booths for each. In one the visitor hears spoken words. which are two numbers, such as "eightsix". The numbers heard are written on the card, and each successive pair is at a lower volume. Twelve pairs of numerals are spoken at successively lower volumes; and then the test is repeated with a different series. In the other type of test, the two numbers are replaced by pure musical tones, each tone being sounded from one to three times, and the listeners write down the number of times they hear the tone. Five tests, each consisting of nine sets of tones at successively lower volumes, are given. The first is a moderately low pitch, 440 cycles per second, which corresponds to $A$ above middle $C$ on the piano. Each following test is one octave higher in pitch and thus the hearing is tested at 440,880, 1,760, 3,520 and 7,040 cycles.

At the San Francisco Exposition were three booths, one arranged for word tests, one for tone tests, and one that could be used for either. At New York eight booths were provided for each type of test. Only the tone tests were recorded. The word tests give only a check on one's ability to understand spoken words, while the tone test, by providing data at five frequencies over the most important part of the aural range, is more suitable for study and analysis. Before a record is made on the test card the attendant puts a mark on it to indicate whether the visitor is male or female, coloured or white, and to which of the five age groups the person belongs, the age groups extending in five groups between 10 and 60. The cards are then run through a Recordak machine which analyses the curves marked on them. The cards are then run through tabulating machines which analyse and sum up the data. The results of this survey indicate a definite falling in hearing acuity with age. This is in harmony with previous data, which indicate a definite falling off in hearing acuity with age, particularly noticeable at the higher frequencies. A rather remarkable fact is that at the low frequencies the falling off with age is less for men than for women, while at the higher frequencies it is less for women than for men. In the lowest age group the difference between men and women is small, but even here the advantage is slightly with the men at low frequencies and with the women at high frequencies. In the age group 50-59 years the difference becomes much more pronounced.

The curves show that for young ears and low frequencies, the tests are grouped fairly closely around the average. For older persons or higher frequencies, the distribution spreads and the number with average hearing becomes small ; most differ from the average in both directions and in varying degrees. The interpretation of the losses at various frequencies needs a specialist, since the evaluation of the effect on one's ability to hear in any particular band of frequencies is a function of many factors. It has been found that one's ability to understand speech can be determined from the average of his hearing losses at 440 , 880 and 1,760 cycles per second as compared with good young ears. If this average is $25 \mathrm{db}$. (decibels), there may be some difficulty in hearing in auditoriums and churches, while if it is $45 \mathrm{db}$. there may be difficulty in hearing in direct conversation. Only if the hearing loss is as much as $65 \mathrm{db}$. will there ordinarily be much difficulty in hearing over the telephone.

By use of these figures, the tests indicate that about one out of twenty-five persons have difficulty in hearing in auditoriums ; one in 125 will have sorne difficulty in direct conversation; and one in 400 over the telephone. Two out of five men between 50 and 59 will have a loss of at least $25 \mathrm{db}$. at 3,520 cycles, while only one in five women will have as great a loss at this frequency. It was found also that about one in 25 of the group from 10 to 19 had a loss of at least $25 \mathrm{db}$. at 7,040 cycles. This figure is important, because it has been found that young people with a hearing loss of this amount will often be found to become progressively worse in later years, but if remedial measures are taken immediately, impairment may be largely checked. No significant difference was found between the visitors to the Fairs who were resident in New York and those resident in San Francisco. The time of day at which each test was taken was marked on the card and permits a comparison of the results for different periods of the day. No consistent difference was found between early morning and late afternoon. There is thus no indication of any effect being produced in the hearing by fatigue. 